\title{
SIMULACIÓN DEL FLUJO DE CAMIONES EN EL PROCESO DE EMBARQUE DE MINERALES PROPUESTO EN EL TERMINAL PORTUARIO DEL CALLAO, PERÚ
}

\author{
Simulation of flow in the process of truck shipment \\ of minerals proposed in Peru Callao port terminal
}

\author{
Luis Ulfe Vega, Edgard Solís Vargas ${ }^{1}$
}

PRESENTACIÓN: MAYO 2016

ACEPTACIÓN: JULIO 2016

\section{RESUMEN}

El presente estudio tiene como finalidad simular las operaciones de embarque de concentrados de mineral en el terminal portuario del Callao, Perú, para dimensionar capacidades y la utilización de cargadores portátiles de alto rendimiento que no requieran apilamiento previo del concentrado sobre la plataforma del Muelle. Dicha simulación permite medir la flota de camiones que hacen el recorrido de los almacenes al muelle y la viabilidad de usar los cargadores portátiles de alto rendimiento para diferentes escenarios.

Palabras clave: simulación, aleatoriedad, puerto, minerales, cargadores portátiles

\begin{abstract}
This study aims to simulate the operations of shipping of mineral extract at Callao Peru port terminal to size the capacities and utilization of high performance portable chargers that do not need previous stacking of the extract on the dock's plataform, this simulation allows to size the truck's fleet (the ones that do the route between the warehouses and the duck) and the viability of using the high performance portable chargers for different scenarios.
\end{abstract}

Keywords: simulation, randomness, port, minerals, portable chargers 


\section{INTRODUCCIÓN}

El estudio de simulación analiza el desempeño del nuevo proceso de embarque de concentrados de minerales a desarrollarse en el muelle No 3 , los mismos que serán transportados desde dos almacenes cercanos al puerto del Callao hasta el mencionado lugar.

Este análisis se realiza a través de un modelo de simulación animado que recrea las operaciones y procesos críticos para el proceso de embarque de minerales, lo que nos permite analizar el desempeño del sistema bajo distintos supuestos, identificar los posibles problemas operativos que se tendrán y conocer la configuración ideal de los equipos que se usarán para esta operación.

El beneficio del estudio radica en que el riesgo de implementar los cambios propuestos en las operaciones descritas previamente sea mínimo, en el sentido de que se contará con un laboratorio virtual, en el que se harán los cambios necesarios para obtener una configuración ideal acorde con las expectativas planteadas, a un costo cero de riesgo con un alto nivel de confiabilidad en los resultados. Ello nos permitirá simular varias operaciones de embarque de mineral bajo diversos supuestos para poder estimar un comportamiento futuro de los procesos de embarque.

La alta variabilidad inherente a los procesos en el embarque de concentrados de minerales, así como la interdependencia que tienen con otros componentes como las operaciones en los almacenes de origen, hace que sean complejos de analizar.

Se presentan las conclusiones obtenidas a partir de la evaluación de los escenarios planteados en el requerimiento de la Autoridad Portuaria Nacional, lo que implica evaluar la utilización de las diversas capacidades y cantidad de cargadores portátiles de alto rendimiento usando uno o dos alimentadores, así como la cantidad de camiones que deberían transportar los concentrados para asegurar un abastecimiento continuo de los concentrados de mineral, lo que permitiría identificar problemas de congestionamiento que se generarían en el flujo.

\section{SIMULACIÓN}

Los encargados de planeamiento muchas veces necesitan manejar numerosas operaciones aleatorias e interdependientes con otros sistemas. Eso implica complejidad de manejo. La necesidad de evaluar el funcionamiento de las operaciones no es satisfecha por ningún sistema de análisis convencional. Los modelos estáticos, como hojas de cálculo o diagramas de flujo, no muestran cómo funciona el sistema dinámicamente en la práctica (Law, 2000).

La simulación es una técnica que consiste en estudiar un sistema real a través de un modelo de computador, el cual refleja los aspectos críticos del proceso analizado. Estos son la variabilidad, la interdependencia entre sus elementos y la dinámica en el tiempo. Todo ello permite evaluar el desempeńo del sistema actual, y facilitar la experimentación de diferentes alternativas de mejora y cambios en los parámetros de operación visualizados en su impacto. Las técnicas analíticas de simulación proveen una poderosa herramienta para procesos de reingeniería, reducción de costos, evaluación de políticas, mejoramiento continuo y otros.

\section{OBJETIVO DEL ESTUDIO}

- Se busca determinar la configuración ideal a implementar para el proceso de embarque de concentrados de mineral que se ejecutará en el muelle nro. 3 del Terminal Portuario del Callao. Esta configuración ideal nos indicará lo siguiente: 
o La capacidad del cargador portátil de alto rendimiento que se utilizará en el proceso de embarque,

o La cantidad de cargadores portátiles de alto rendimiento que se utilizará en el proceso de embarque,

o La cantidad de alimentadores tipo «Samson» que deben usar los cargadores portátiles de alto rendimiento.

- La configuración ideal buscará que el proceso de embarque de los minerales se realice en el menor tiempo posible, desde que comienza el embarque hasta que finaliza. A este tiempo transcurrido desde el inicio del embarque hasta la finalización del mismo lo llamaremos «horas transcurridas para el embarque».

- La configuración ideal identificará los posibles cuellos de botella que se generen por falta de capacidad para el estacionamiento de los camiones y, por ende, determinará la cantidad de camiones que deberían trabajar en esta operación.

\section{SUPUESTOS Y CONSIDERACIONES DEL MODELO DE SIMULACIÓN}

El modelo de simulación ha sido desarrollado bajo los siguientes límites y supuestos acordes a lo estipulado en el requerimiento de la Autoridad Portuaria Nacional:

- El modelo de simulación considera solamente el proceso de embarque de concentrados de mineral por el amarradero nro. 3B.

- Estimamos que los camiones ingresarán por la puerta nro. 5 del Terminal Portuario del Callao, que es para uso exclusivo de ese transporte. En el caso de usar dicho acceso para el ingreso de otro tipo de unidades como contenedores, será necesario realizar otro análisis adicional no contemplado en el presente estudio (Instituto de Consultoría S.A. y consultora Cardenas y Bautista, 2004).

- La información usada en el simulador respecto a los tiempos requeridos para las maniobras de descarga de los camiones en los cargadores portátiles de alto rendimiento se ha realizado asumiendo los tiempos de las operaciones de carga de minerales en el Terminal Portuario de Salaverry.

- Los procesos en los almacenes de concentrados de mineral que abastecen al Terminal Portuario del Callao, representados en el simulador, son aquellos que se relacionan exclusivamente con el carguío de camiones con concentrados para ser transportados hasta el muelle 3B. Las operaciones adicionales que se ejecutan en estos almacenes no son materia de análisis y no han sido consideradas, por ejemplo, el proceso de descarga de los camiones que llegan desde las minas a dicho almacén.

- La cola de estacionamiento de los camiones (junto a la puerta de ingreso 5) tiene una capacidad de 13 camiones si consideramos un espacio disponible aproximado de 250 metros para la cola y que cada camión requiere 18.5 metros aproximadamente.

- La capacidad máxima de la cola de camiones previa al ingreso de camiones por la puerta 5 se ha estimado en cinco (ver gráfico 1). 


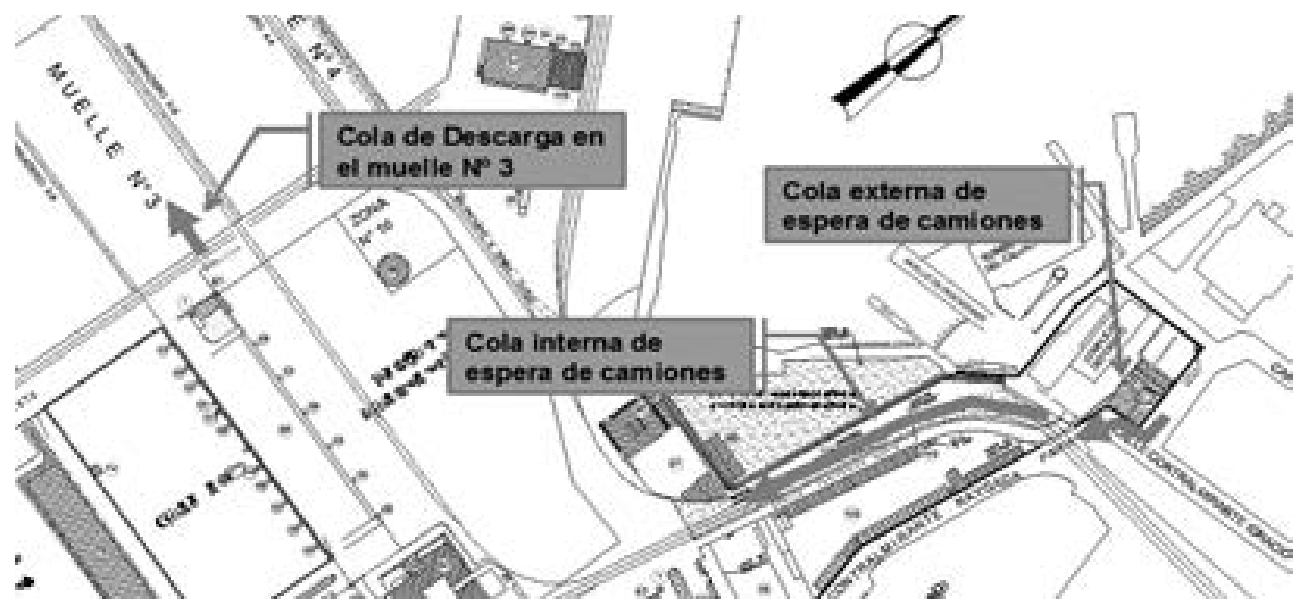

Gráfico 1. Ubicación de las colas de camiones en el T. P. Callao. Fuente: (Autoridad Portuaria Nacional - $A P N)$

\section{PRINCIPALES VARIABLES DE DECISIÓN}

Gracias al análisis del sistema bajo estudio, se determinó que la principal variable de interés es el tiempo requerido para realizar el embarque de minerales. Este tiempo se considera desde que comienza el fletamento hasta que finaliza.

Otros factores a considerar para medir el desempeño del sistema son los siguientes:

- Las capacidades de las colas en las diversas etapas del flujo,

- El número de cargadores portátiles y su rendimiento,

- El número de alimentadores por cargador,

- La cantidad de camiones de 30 toneladas,

- La tasa de toneladas embarcadas por hora,

- Los cuellos de botella en los flujos de abastecimiento.

\section{EL FLUJO DEL PROCESO MODELADO}

El modelo de simulación construido representa el siguiente flujo de actividades para ejecutar el embarque de concentrados de mineral (ver gráfico 2).

En el caso de que el buque ya esté posicionado y se hayan dado los pasos previos para comenzar con el proceso de embarque de los concentrados de minerales, se presenta el diagrama de flujo de los procesos representados en el modelo de simulación. Este proceso muestra un nivel general, pero, en el modelo de simulación, se han desagregado las actividades críticas para analizar su impacto en el proceso de embarque (Instituto de Consultoría S.A. y consultora Cardenas y Bautista, 2004).

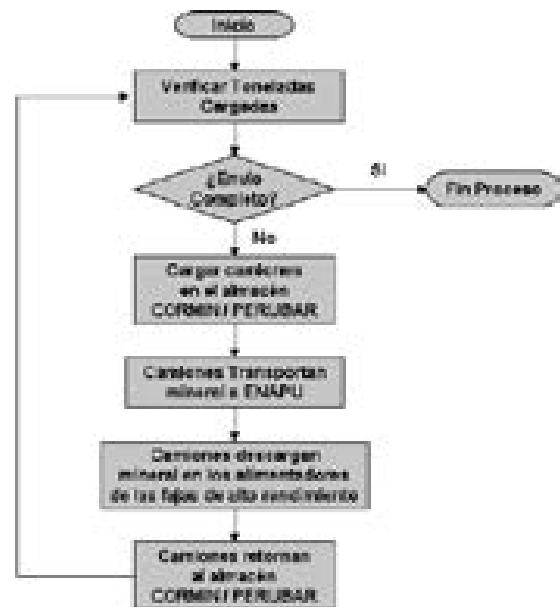

Gráfico 2. Flujo del proceso resumido.

Fuente: generación propia. 


\section{EL MODELO DE SIMULACIÓN}

El modelo de simulación ha sido construido usando el software de simulación PROMODEL, que toma como guía el diagrama de flujo mostrado anteriormente (Harrel, Bateman et al., 2005).

También se usa un plano en AUTOCAD de las instalaciones del T. P. del Callao (proporcionado por la Gerencia de Ingeniería y Mantenimiento de la Empresa Nacional de Puertos -ENAPU) para ser usado como fondo del modelo y se trabaja a escala con él, lo que permite visualizar las distancias y movimientos acordes a la escala del plano.

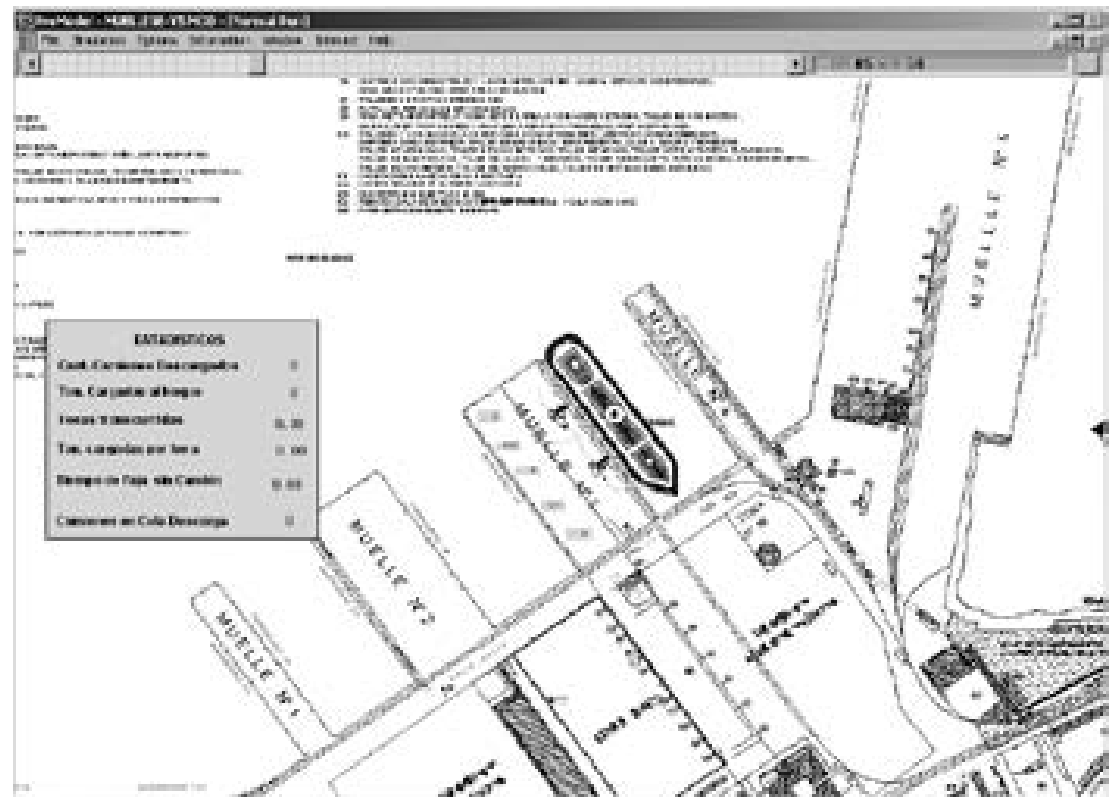

Gráfico 3. Vista parcial del modelo de simulación del Muelle nro. 3- T. P. del Callao. Fuente: (Empresa Nacional de Puertos - ENAPU).

\section{INFORMACIÓN ESTADÍSTICA USADA EN EL MODELO DE SIMULACIÓN}

El modelo de simulación requiere de información estadística para representar la aleatoriedad real del sistema. Para esto, se ha recopilado información de demoras de actividades y desplazamientos, las mismas que se procesaron utilizando el módulo estadístico de datos de ProModel Stat::Fit (www. promodel.com). Los datos que fueron recolectados y analizados son los que siguen:

- El tiempo de demora de los camiones al ingresar a los almacenes alimentadores externos al T. P. del Callao,

- El tiempo de demora de los mismos para estacionarse adecuadamente antes de comenzar la carga,

- El tiempo de demora de carga de camiones en los almacenes,

- El tiempo que los camiones demoran en la puerta de salida de los almacenes, lo cual involucra las demoras producto del lavado de los camiones antes de salir del almacén,

- La demora de transporte desde los almacenes a la puerta de acceso nro. 5 del T. P. del Callao,

- El tiempo de atención de camión en la puerta de ingreso nro. 5 del T. P. del Callao, 
- El tiempo de desplazamiento de los camiones dentro de las instalaciones del T. P. del Callao, que considera una velocidad de $10 \mathrm{~km} / \mathrm{h}$., en el que se consignan las distancias reales de acuerdo al plano en Autocad,

- El tiempo requerido para que los camiones se ubiquen adecuadamente antes de comenzar a descargar,

- El tiempo de descarga de los camiones en los alimentadores de los cargadores portátiles de alto rendimiento,

- El tiempo requerido para que el camión se retire de la ubicación de descarga y permita aproximarse al siguiente camión,

- El tiempo requerido por el camión para dirigirse a la puerta de salida nro. 5 del T. P. del Callao,

- El tiempo de atención de camiones en la puerta de salida nro. 5 del T. P. del Callao,

- El tiempo de demora en el recorrido desde el T. P. del Callao hasta los almacenes alimentadores.

A continuación, se presentan algunos ejemplos de funciones de probabilidad obtenidas de la data muestreada en campo:

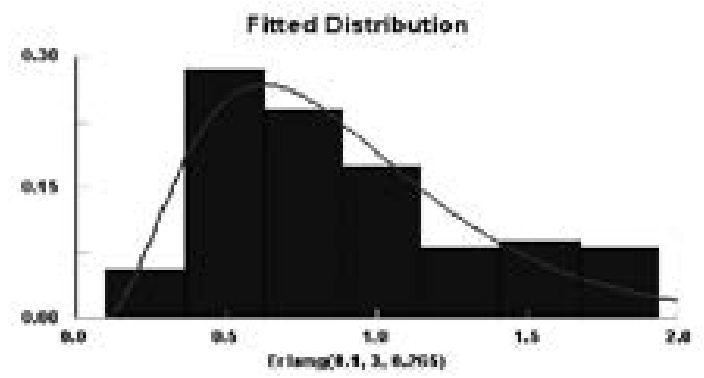

Gráfico 4. Tiempo de atención de camiones en la Puerta de Ingreso nro. 4 del T. P. Callao.

Fuente: generación propia.

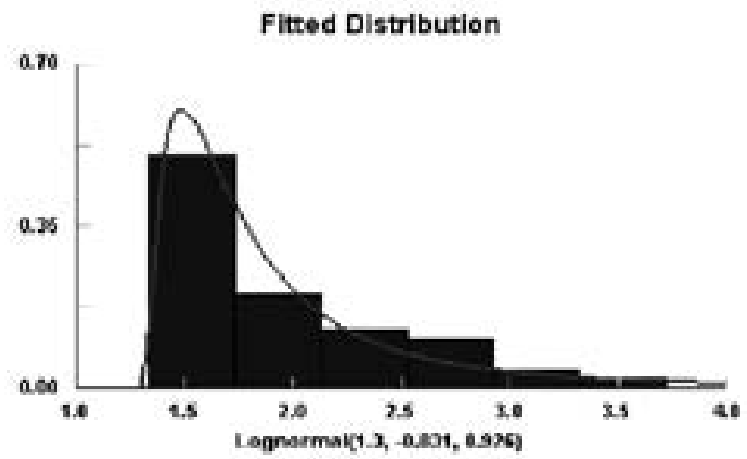

Gráfico 5. Tiempo de carguio de mineral en almacén. Fuente: generación propia.

\section{PRINCIPALES ESCENARIOS EVALUADOS}

A continuación, mostramos un resumen que compara las mejores configuraciones halladas: 


\begin{tabular}{|c|c|c|c|c|c|c|c|c|}
\hline & \multicolumn{2}{|c|}{ FSOFNARIO 1} & \multicolumn{2}{|c|}{ ESCEMARID? } & \multicolumn{2}{|c|}{ ESCEMARIO] } & \multicolumn{2}{|c|}{ FSCEMARID 4} \\
\hline & COFMiK & PEFUAAR & CORMIN & PERLBAR & CORGIN & PFRUBAR & CORMIR & PEPUBARE \\
\hline Cantidad de Cargadoles & 1 & 1 & 1 & 1 & 2 & 2 & 2 & 2 \\
\hline Alimentadores por carqador & 1 & 1 & 2 & 2 & 1 & 1 & 2 & 3 \\
\hline Purtos de carga en almocen & 1 & 1 & 1 & 1 & 1 & 1 & 1 & \\
\hline Carnianes Dsades & 10 & 13 & 13 & 20 & 15 & 20 & 15 & 2il \\
\hline Carniones Descargades & 567 & 567 & 6) & 667 & 657 & 657 & 667 & $66 r$ \\
\hline Ionelados c & 70.010 & 20.010 & 20.010 & 20010 & 20010 & 20010 & 20.010 & 20.010 \\
\hline Heras Tramscuerides & 12.1 & 422 & 217 & $x 7.7$ & 27.7 & 26.9 & 27.7 & 27.3 \\
\hline Toneladas catacad $\mathrm{p}$ & 975.2 & 174.1 & 723.3 & 723.4 & 723.6 & 713,2 & 721.3 & 733.3 \\
\hline Camiones Cargados & 667 & 667 & 67 & 667 & 647 & 667 & 667 & 667 \\
\hline
\end{tabular}

Tabla 1. Resultados de los mejores escenarios considerando UN punto de carga en los almacenes de CORMIN y PERUBAR.

\subsection{El mejor escenario con un punto de carga en los almacenes}

Podemos notar que el escenario 2 tiene un mejor desempeño, ya que tiene como configuración un cargador móvil de alta velocidad y dos alimentadores tipo «Samson». La cantidad de horas requeridas para embarcar aproximadamente 20,000 toneladas de concentrado de mineral es como sigue:

- Alrededor de 27.7 horas considerando que toda la carga sea transportada desde el almacén de Cormin usando 15 camiones (se evaluaron en el rango de 10 a 20 camiones).

- Alrededor de 27.7 horas considerando que toda la carga sea transportada desde el almacén de Perubar usando 20 camiones (se evaluaron en el rango de 10 a 30 camiones).

\begin{tabular}{|c|c|c|c|c|c|c|c|c|}
\hline & \multicolumn{2}{|c|}{ ESCFNAADO 1} & \multicolumn{2}{|c|}{ FSCFIIARDO 2} & \multicolumn{2}{|c|}{ ESCENAALO 3} & \multicolumn{2}{|c|}{ FSCENARIO 4} \\
\hline & CORNII & PERUIIAR & CORMits & PEFUTAR & CORMA & PERUHAR & CORAM & PERUERR \\
\hline Cantisad do Cargadores & 1 & 1 & 1 & 1 & 2 & 2 & 2 & $z$ \\
\hline Allmentaderes pol cargador & 1 & 1 & 2 & 2 & 1 & 1 & 2 & 8 \\
\hline Puntes de carga en aimacén & 2 & 2 & 2 & 2 & 2 & 2 & 2 & 2 \\
\hline Camilanes Usates & 10 & 12 & 20 & 28 & 20 & 22 & 죠 & 28 \\
\hline 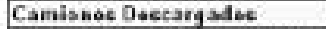 & 5 & 667 & C67 & $\alpha G$ & C6.] & [6] & $\cos$ & Q.5 \\
\hline Tenelades carzadas ol baque & 20.210 & 20.010 & 20,010 & 20,010 & 20,010 & 20,010 & 20,010 & 20.016 \\
\hline Hofac Transcturidas & 123 & 12.1 & 202 & 213 & 21.3 & 21.3 & 102] & 20.5 \\
\hline Toneladas carjadas por hora & 6745 & 474.9 & 90.7 & 937.3 & 916.2 & 945.3 & 1130.1 & 971.8 \\
\hline Camishes Catgodos & इ6] & 657 & Bका & Eका & (6) & [6] & हE) & $6 \%$ \\
\hline
\end{tabular}

Tabla 2. Resultados de los mejores escenarios considerando DOS puntos de carga en los almacenes de Cormin y Perubar

\subsection{El mejor escenario con dos puntos de carga en los almacenes}

Podemos notar que el escenario 4 tiene un mejor desempeño, ya que tiene como configuración dos cargadores móviles de alta velocidad y dos alimentadores tipo «Samson». La cantidad de horas requeridas para embarcar aproximadamente 20,000 toneladas de concentrado de mineral es la siguiente:

- Alrededor de 13.9 horas considerando que toda la carga sea transportada desde el almacén de Corbin usando 30 camiones (se evaluaron escenarios para varios niveles de camiones).

- Alrededor de 20.6 horas considerando que toda la carga sea transportada desde el almacén de Perubar usando 25 camiones (se evaluaron escenarios para varios niveles de camiones). 


\section{CONCLUSIONES}

Las conclusiones a las que llegamos, luego de analizar 160 escenarios o variaciones en la configuración, fueron que hallamos las mejores configuraciones considerando uno y dos puntos de carga en los almacenes de origen, las mismas que describimos a continuación.

\subsection{Considerando un punto de carga en los almacenes de origen (mejor escenario 2)}

Con esta configuración logramos los siguientes resultados para diversos tonelajes a cargar, considerando los almacenes de origen:

\subsubsection{Evaluación del escenario 2 con diversos tonelajes a cargar}

\begin{tabular}{|c|c|c|c|c|c|}
\hline EVALUACIÓN ESCENARIO 2 & & & & & \\
\hline \multicolumn{6}{|l|}{ Almacén de origen: Cormin } \\
\hline Toneladas cargadas al buque & 5,000 & 10,000 & 15,000 & 20,000 & 25,000 \\
\hline Cantidad de cargadores & 1 & 1 & 1 & 1 & 1 \\
\hline Alimentadores por cargador & 2 & 2 & 2 & 2 & 2 \\
\hline Puntos de carga en almacén & 1 & 1 & 1 & 1 & 1 \\
\hline Camiones usados & 15 & 15 & 15 & 15 & 15 \\
\hline Camiones descargados & 167 & 334 & 501 & 667 & 834 \\
\hline Horas transcurridas & 7.0 & 14.0 & 21.0 & 27.7 & 34.7 \\
\hline Toneladas cargadas por hora & 711.4 & 716.0 & 715.1 & 722.8 & 722.1 \\
\hline Camiones cargados & 167 & 334 & 501 & 667 & 834 \\
\hline
\end{tabular}

\begin{tabular}{lccccc}
\multicolumn{1}{c}{ Almacén de origen: Perubar } \\
\multicolumn{1}{c}{ Toneladas cargadas al buque } & 5,000 & 10,000 & 15,000 & 20,000 & 25,000 \\
\hline Cantidad de cargadores & 1 & 1 & 1 & 1 & 1 \\
\hline Alimentadores por cargador & 2 & 2 & 2 & 2 & 2 \\
\hline Puntos de carga en almacén & 1 & 1 & 1 & 1 & 1 \\
\hline Camiones usados & 20 & 20 & 20 & 20 & 20 \\
\hline Camiones descargados & 167 & 334 & 501 & 667 & 834 \\
\hline Horas transcurridas & 7.0 & 14.0 & 20.8 & 27.7 & 34.5 \\
\hline Toneladas cargadas por hora & 711.5 & 716.5 & 723.3 & 723.4 & 724.6 \\
\hline Camiones cargados & 167 & 334 & 501 & 667 & 834 \\
\hline
\end{tabular}

Tabla 3. Escenario 2 con diversos niveles de tonelaje a cargar.

\subsubsection{Cantidad de camiones a utilizar}

Estos resultados son el punto de equilibrio mínimo. Se recomienda que los almacenes destinen una cantidad de camiones de acuerdo a la siguiente configuración:

Cormin: 15 camiones de 30 toneladas.

Perubar: 20 camiones de 30 toneladas. 


\subsubsection{Capacidad requerida del cargador}

De acuerdo a los resultados obtenidos por el simulador, el cargador móvil de alta velocidad debe tener una capacidad superior a las 800 toneladas. Si se consideran las especificaciones técnicas del fabricante B\&W Mechanical Handling Ltd., se trataría de un cargador móvil con una capacidad de 1200 TPH (http://www.samson-mh.com/).

\subsubsection{Cantidad de alimentadores a utilizar}

El análisis de simulación nos indica que se requieren dos alimentadores que trabajen en simultáneo y ubicados en forma paralela al buque, ambos en el mismo sentido en forma de ele con respecto al cargador, tal como se muestra en el siguiente gráfico.

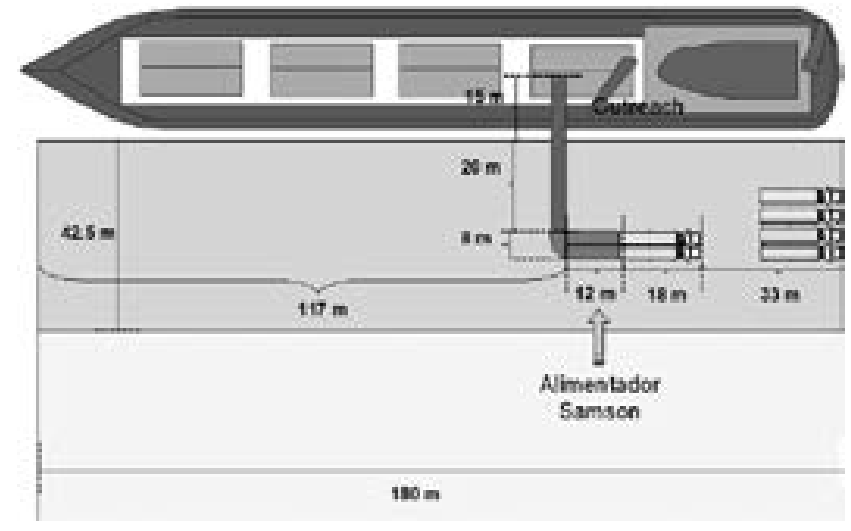

Gráfico 6. Disposición física del cargador y los alimentadores. Fuente: generación propia.

\subsection{Considerando dos puntos de carga en los almacenes de origen (mejor escenario 4)}

Con esta configuración, logramos los siguientes resultados para diversos tonelajes a cargar considerando los almacenes de origen.

\subsubsection{Evaluación del escenario 4 con diversos tonelajes a cargar}

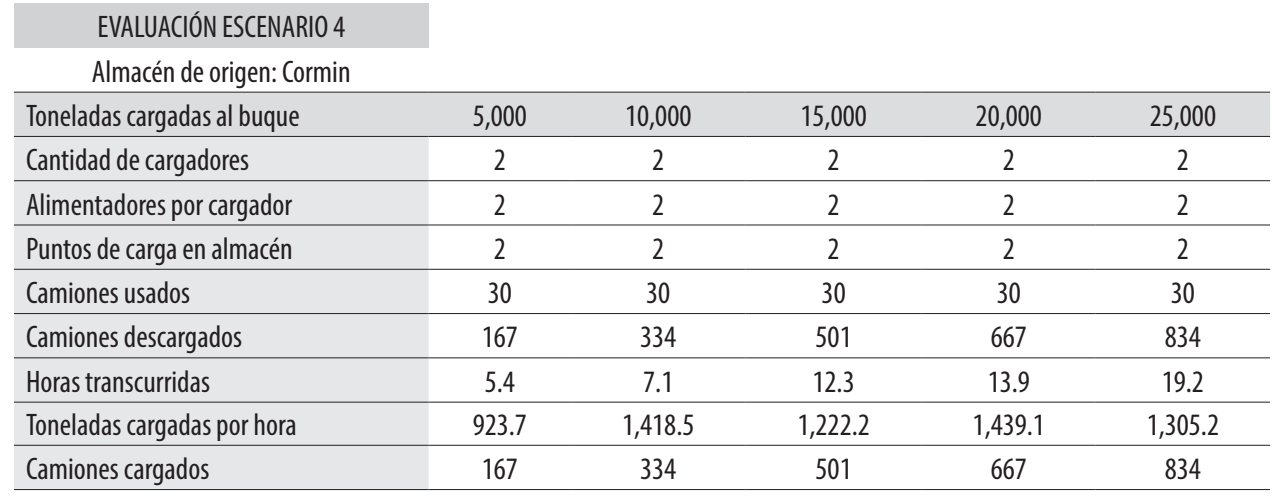


Almacén de origen: Perubar

\begin{tabular}{lccccc}
\hline Toneladas cargadas al buque & 5,000 & 10,000 & 15,000 & 20,000 & 25,000 \\
\hline Cantidad de cargadores & 2 & 2 & 2 & 2 & 2 \\
\hline Alimentadores por cargador & 2 & 2 & 2 & 2 & 2 \\
\hline Puntos de carga en almacén & 2 & 2 & 2 & 2 & 2 \\
\hline Camiones usados & 25 & 25 & 25 & 25 & 25 \\
\hline Camiones descargados & 167 & 334 & 501 & 667 & 834 \\
\hline Horas transcurridas & 5.5 & 10.4 & 15.6 & 20.6 & 25.9 \\
\hline Toneladas cargadas por hora & 909.1 & 966.0 & 962.0 & 971.8 & 965.1 \\
\hline Camiones cargados & 167 & 334 & 501 & 667 & 834 \\
\hline
\end{tabular}

Tabla 4. Escenario 4 con diversos niveles de tonelaje a cargar

\subsubsection{Cantidad de camiones a utilizar}

Estos resultados son el punto de equilibrio mínimo. Se recomienda que los almacenes destinen una cantidad de camiones, de acuerdo a la siguiente configuración:

Cormin: 30 camiones de 30 toneladas.

Perubar: 25 camiones de 30 toneladas.

\subsubsection{Capacidad requerida del cargador}

El análisis de simulación nos indica que se requieren dos cargadores móviles de alta velocidad, cada uno con dos alimentadores que trabajen en simultáneo.

\subsubsection{Cantidad de alimentadores a utilizar}

El análisis de simulación nos indica que se requieren dos alimentadores que trabajen en simultáneo y ubicados en forma paralela al buque, ambos en el mismo sentido en forma de ele con respecto al cargador, tal como se muestra en el siguiente gráfico.

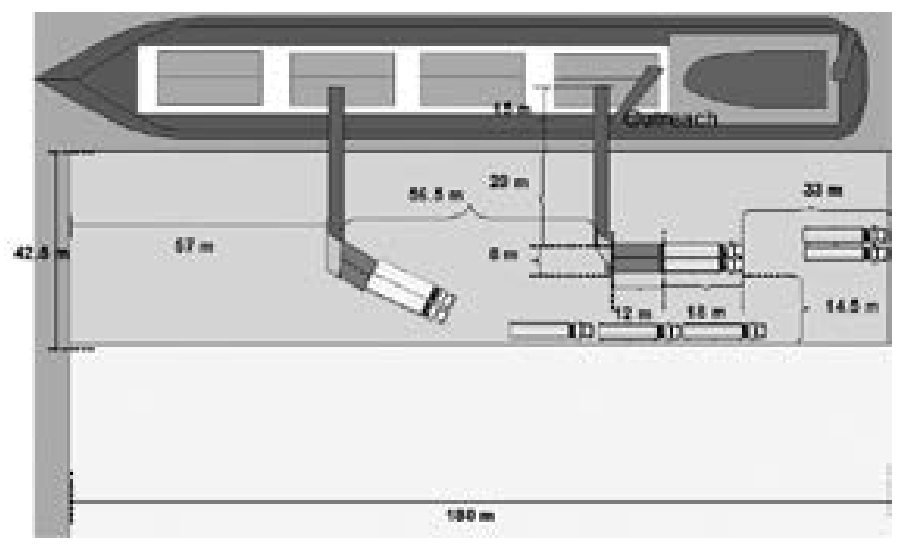

Gráfico 6. Disposición física del cargador y los alimentadores. Fuente: generación propia. 


\section{MEJORA EN LOS ALMACENES DE ORIGEN}

En los almacenes de origen, notamos que, normalmente usan un punto de carga de camiones, dependiendo del espacio disponible. El modelo nos muestra que la velocidad de aprovisionamiento de los camiones en el muelle nro. 3 es altamente sensible a esta cantidad de puntos de carga.

\section{RECOMENDACIONES}

- La configuración (faja móvil, alimentador, camiones) que se elija debe cumplir las dimensiones mínimas de espacio de seguridad para el correcto funcionamiento del proceso de embarque en el muelle nro. 3. El presente estudio de simulación no involucra el nivel de seguridad en las operaciones del muelle; únicamente se planteó diversas configuraciones posibles considerando las dimensiones del fabricante del cargador móvil de alta velocidad, el alimentador y la posición del vehículo.

- Se sugiere un estudio de las estructuras del muelle nro. 3 para verificar si está en condiciones de resistir las operaciones de embarque de mineral por el Amarradero nro. 3B, de acuerdo a la configuración que sea elegida, la cual deberá tener en cuenta el peso de la(s) faja(s) móvil(es), el(los) alimentador(es) y la pequeña cola de camiones en espera por la descarga que se forme en el muelle.

\section{REFERENCIAS BIBLIOGRÁFICAS}

[1] Harrel, C. R. y Tumay, K. (1995) Simulation made easy. A manager's guide (1a ed.). USA: Industrial and Management Press.

[2] Harrel, C. R., Bateman, R. y otros. (2005). System Improvement using Simulation (5a ed.). USA: Promodel Corporation.

[3] Instituto de Consultoría S.A. y consultora Cardenas y Bautista. (2004). Estudio para el Desarrollo de la Ingeniería Básica del Proyecto de Recepción, Transporte y Embarque de Concentrados por el Muelle No 5 del Terminal Portuario del Callao. Perú: (s. e.).

[4] Law, A. M. y Kelton, D. (2000). Simulation Modeling \& Analysis (3a ed.). USA: Ed. McGraw-Hill.

[5] Promodel Corporation. (2008). Promodel Corp. User Guide. USA: (s. e.).

\section{Referencias en la Web}

http://www.promodel.com/

http://www.samson-mh.com/ 